\title{
The Influence of Allowable Refill Gaps on Detecting Long-Term Opioid Therapy: An Analysis of Population-Based Administrative Dispensing Data Among Patients with Knee Arthritis Awaiting Total Knee Arthroplasty
}

\author{
C. Michael Goplen, MD; Jason R. Randall, PhD; Sung Hyun Kang, MSc; Fatemeh Vakilian, MSc; \\ C. Allyson Jones, PT, PhD; Donald C. Voaklander, PhD; and Lauren A. Beaupre, PT, PhD
}

\begin{abstract}
BACKGROUND: It is challenging to detect long-term opioid therapy (LTOT) using administrative data, as refill gaps can disrupt opioid utilization episodes. Previous studies have used various methods to define LTOT and allowable refill gaps with little supporting evidence.

OBJECTIVE: To describe the effect of allowable refill gaps on detecting LTOT among a cohort of patients with arthritis awaiting total knee arthroplasty (TKA) using 3 different methods.
\end{abstract}

METHODS: A retrospective analysis of multicenter population-based data between January 1, 2012, and December 31, 2016, identified patients prescribed opioids before TKA in Alberta, Canada. We described 3 methods to detect LTOT based on a (1) fixed number of days between prescriptions; (2) fraction of the preceding prescription length; and (3) combination method that selected whichever refill gap was greatest. We then compared the number of patients classified as long-term opioid users by varying the number of days between prescriptions from 1-90 days (fixed method) or 0.04-3.2 times the duration (fraction method) for each method and refill gap.

RESULTS: Of the 14,252 patients included in our cohort, 4,393 patients (31\%) had an opioid prescription within 180 days before TKA. Detection of LTOT varied from $4.4 \%$ to $14.6 \%$ (fixed method), $4.2 \%$ to $13.2 \%$ (fraction method), and $4.5 \%$ to $15.1 \%$ (mixed method) as refill gaps varied from minimum to maximum. As refills gaps increased, the dose and duration of opioids in the utilization episode decreased for all 3 methods.

CONCLUSIONS: The allowable refill gap between opioid prescriptions can influence the estimated rate of LTOT when using administrative pharmaceutical dispensing data. Definitional parameters should be carefully considered when using administrative data to define consistent opioid use.

J Manag Care Spec Pharm. 2019;25(10):1064-72

Copyright $\odot 2019$, Academy of Managed Care Pharmacy. All rights reserved.

\section{What is already known about this subject}

Continuous medication users often have small gaps between prescription dispensings, referred to as refill gaps.

Multiple methods have been described to calculate allowable refill gaps to define consistent medication use with pharmaceutical administrative dispensing data.

\section{What this study adds}

The allowable refill gap can substantially change the estimated rate of long-term opioid use and influence clinical outcomes.

Fixed, fraction, and mixed methods provide similar estimates of consistent opioid use at comparable refill gap thresholds.

( ver the past 2 decades, the number of opioids prescribed for chronic noncancer pain conditions, such as arthritis, has dramatically increased..$^{1-5}$ These prescribing patterns are thought to be responsible for the increased prevalence of patients prescribed long-term opioid therapy (LTOT); that is, "daily or near-daily use of opioids for at least 90 days, often indefinitely." ${ }^{\text {-8 }}$ LTOT among patients with symptomatic arthritis has gained substantial clinical and research interest as both Canadian and American opioid prescribing guidelines now suggest a much more limited, if any, role of opioids for these patients. ${ }^{9-11}$ These recommendations are based on the accumulating evidence that suggests opioids provide no improvement in pain or function when compared to acetaminophen or ibuprofen but have increased rates of adverse events. $^{12,13}$

LTOT before surgery has also been associated with increased rates of postoperative complications and worse patient outcomes after elective surgery. ${ }^{14-16}$ For instance, a recent metaanalysis reported that patients with hip or knee arthritis prescribed opioids before surgery had worse patient-reported outcomes after elective total joint arthroplasty. ${ }^{17}$ However, the prevalence of LTOT in North America for this patient population is still unclear, as rates are not only dependent on regional prescribing practices but are also affected by methodological parameters used to detect LTOT. ${ }^{18-22}$

Pharmaceutical administrative databases containing medication-dispensing records from community pharmacies are routinely used to analyze opioid prescriptions in Canada and the United States, as data are readily available for large numbers of patients and are relatively inexpensive to access. ${ }^{1,21,23-25}$ However, it can be challenging to identify 
consistent opioid utilization episodes that meet the threshold for LTOT, since any gaps between contiguous prescriptions disrupt the classification episode. ${ }^{26-28}$ These gaps are a result of inconsistent prescription refill patterns, where there may be a short period between the previous prescriptions calculated end date and the dispensing date of the subsequent prescription. ${ }^{27}$ A grace period, referred to as an allowable refill gap, is often used to analyze these pharmaceutical-dispensing records, since patients appear to consistently use the prescribed medication despite small gaps between prescriptions..$^{21,27,28}$

In other clinical areas, there has been significant interest to describe methods that account for allowable refill gaps, and the different influence that these methods can have on estimating consistent medication use. ${ }^{27,28}$ Current LTOT research uses arbitrary allowable refill gaps thresholds, with little understanding of how different methods and refill gaps might influence the estimated rates of LTOT or patient outcomes. ${ }^{20,23,29,30}$

The primary objective of this study was to describe the influence of allowable refill gaps on estimating LTOT among a cohort of patients with knee arthritis awaiting total knee arthroplasty (TKA). Our secondary objectives were to determine the influence of allowable refill gaps on patient-reported daily opioid dose, mean opioid episode duration, and patient outcomes after TKA.

\section{Methods}

\section{Study Design}

A retrospective review of individual-level data identified patients who underwent primary, elective unilateral TKA between January 1, 2013, and December 31, 2015, in Alberta, Canada. This patient cohort was selected because it was thought to represent a stable population of patients with chronic noncancer pain secondary to end-stage knee arthritis, as confirmed by their requirement for TKA. Canada's socialized health care system does not restrict access to TKA, but patients wait on average 6 months for surgery. ${ }^{31}$ Patients who underwent a subsequent contralateral TKA were included only once for whichever procedure occurred first. Patients who underwent simultaneous bilateral TKA or revision TKA within 1 year of the index procedure were excluded from the analysis. The Strengthening the Reporting of Observational Studies in Epidemiology (STROBE) guidelines were followed in this observational study. ${ }^{32}$ The study protocol was approved by the research ethics board at the University of Alberta, Edmonton, Alberta (Pro00076296).

\section{Data Sources}

Surgical data were obtained from the Alberta Bone and Joint Health Institute. This database contains patient demographic information, procedure details, and clinical outcomes after TKA. Opioid-dispensing data were obtained from the Pharmaceutical Information Network (PIN), a provincial pharmaceutical repository that maintains individual-level pharmacotherapy records and dispensing information from all community pharmacies in Alberta, based on the province's universal health insurance program. Because Canada has universal health care coverage, these databases represent population-based dispensing practices within each Canadian province.

A record was created in PIN each time a medication was dispensed from a community pharmacy in Alberta and contained the drug information number, anatomic therapeutic code, date dispensed, dose, and duration (days supplied). Each PIN entry was linked to a patient's Unique Lifetime Identifier (ULI), a unique number assigned to all persons who receive health services in Alberta. Each patient's PIN profile was queried for the 180 days before the index surgery to determine their opioid-dispensing history. The end date for each dispensing was then calculated by adding the duration to the dispensing date for each PIN entry. All datasets were deterministically linked using patient ULIs that were previously scrambled with an algorithm that deidentified each ULI but still preserved the ability to link across datasets.

We determined the allowable refill gap between opioid prescriptions by 3 different methods (Figure 1). First, we determined the allowable refill gap between prescriptions using a fixed days method. ${ }^{27,33}$ This method sets a maximum number of days allowed between the end of one prescription and the start date of the next recorded prescription, which is independent of the length of prescriptions being analyzed. We varied the number of days from 1 to a maximum of 90 days. The upper limit of 90 days was chosen as this guarantees that each patient would have a least 1 day of opioid prescription if classified an opioid user (OU) in the 180-day window. The 180-day opioid-free period had been previously used in studies investigating LTOT and is the established threshold for opioid discontinuation. ${ }^{23}$

Second, we applied a fraction method to determine the allowable refill gap between prescriptions. ${ }^{27}$ This method used the length of the preceding prescription duration to determine the following allowable refill gap. We varied the fraction incrementally from 0.04-3.2. Previous work investigating cardiovascular medication adherence aligned 90-day prescriptions with a fraction of 1 , since this was the maximum amount of days that health insurance would compensate pharmacies. ${ }^{27}$ In our study, we aligned the fraction and fixed methods based on Canadian opioid guidelines that suggest opioid prescriptions be limited to less than 28 days. ${ }^{9}$ Therefore, a fraction gap of 1 was aligned to a fixed refill gap of 28 days.

In addition, a third method (mixed method) was developed that combined the fixed and fraction methods and defaulted to the largest refill gap calculated (Figure 1). 


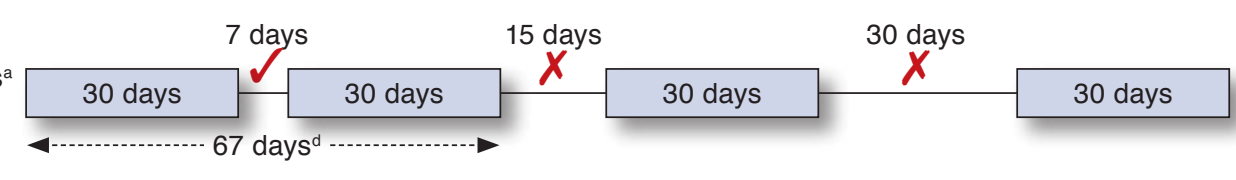

Fraction (0.25): 7.5 days $^{a}$ Fixed: 7 days

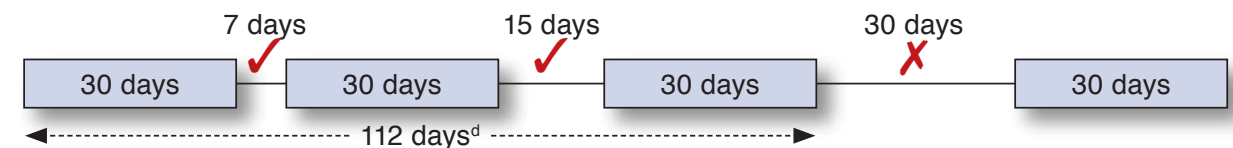

Fraction: Long-term Fixed: Intermittent Fraction (0.5): 15 days $^{\mathrm{b}}$ Fixed: 14 days

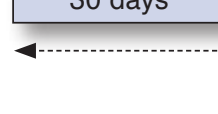

Fraction (0.5): 30 days $^{\mathrm{c}}$ Fixed: 14 days

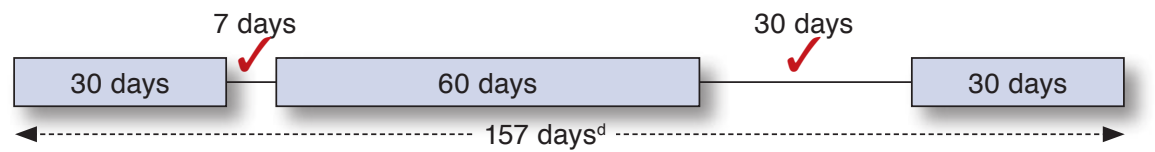

157 days $^{d}$

Continuation of opioid utilization episode

$X$ Maximum allowable refill gap exceeded and opioid utilization episode

${ }^{a}$ Calculated using the preceeding prescription length of 30 days and a fraction of 0.25 (30 days $\times 0.25=7.5$ days).

${ }^{b}$ Calculated using the preceeding prescription length of 30 days and a fraction of 0.5 (30 days $\times 0.5=15$ days).

${ }^{c}$ Calculated using the preceeding prescription length of 60 days and a fraction of 0.5 (60 days $\times 0.5=30$ days).

dTotal opioid episode duration calculated based on mixed methods.

\section{Primary Outcome: Long-Term Opioid Use}

Long-term OUs were defined as patients who had 90 days or more of continuous opioid dispensings within 180 days before TKA; this group was our primary outcome of interest. These parameters were consistent with the definition of LTOT. Intermittent OUs had recorded opioid dispensings within the 180 days before TKA but did not meet the threshold parameters for a long-term OUs. Opioid-naive patients did not have a recorded opioid dispensing within 180 days before TKA and were not included in the refill methods analysis. The allowable refill gap was dependent on the specific method (fraction, fixed, or mixed).

\section{Secondary Outcomes}

Opioid Utilization Episode Duration. The duration of a patient's opioid utilization episode was determined by the difference between the first opioid-dispensing date and the end date of the last continuous opioid prescription. The continuous opioid utilization episode terminated if the gap between the end date of the preceding prescription and the start date of the next opioid prescription exceeded the allowable refill gap or a surgical date was encountered (Figure 1). Therefore, the total days in the opioid utilization episode contained both the number of active days a patient held an opioid prescription and the allowable refill gap (days) between prescriptions (Figure 1). If patients had multiple prescriptions, then the dispensation date and days supplied were used to adjust the end date of the prescriptions for each subsequent dispensation. If 2 or more overlapping prescriptions with the same dispensing date and days supplied were encountered, the prescriptions beyond the first would not extend the length of the episode but would count toward the total dosage morphine equivalent dose (MED) received. If a short prescription was received within a longer prescription (where the new prescription's supply days did not extend past the predicted end of the previous prescription), then the total dosage (MED) was added to the treatment course, but the end date of the prescription course was not adjusted. In this instance, the fraction method would also continue to use the fraction of the previous, longer prescription. If a prescription was expected to end beyond the currently estimated end date, then a new end date was estimated using the dispensation date and total days supplied of the prescription, and the fraction days were also adjusted to match the fraction of this prescription.

Daily Morphine Equivalent Dose. Patients' individual opioid dispensings were converted to a daily MED by multiplying the daily dose (mg) by the corresponding MED. ${ }^{34}$ Morphine conversion factors were based on established conversion opioid factors. ${ }^{9}$ The daily dose of opioid was calculated by dividing the total MED for the opioid utilization episode by the corresponding duration of the episode as calculated by the fixed, fraction, or mixed method. 


\begin{tabular}{|c|c|}
\hline \multicolumn{2}{|c|}{$\begin{array}{l}\text { Characteristics of the Study Cohort and } \\
\text { Opioid Prescriptions }\end{array}$} \\
\hline \multicolumn{2}{|l|}{ Variable } \\
\hline Patients (n) & 14,252 \\
\hline Mean age (SD) & $66.8 \quad(9.3)$ \\
\hline Female, n (\%) & $8,608(60.4)$ \\
\hline Total opioid prescriptions (n) & 17,617 \\
\hline Individual opioid prescription duration, median days (IQR) & $24(21.0)$ \\
\hline \multicolumn{2}{|l|}{ Type of opioid prescriptions, $\mathrm{n}(\%)$} \\
\hline Codeine & $6,755(38.3)$ \\
\hline Tramadol & $4,203(23.9)$ \\
\hline Oxycodone & $2,836(16.1)$ \\
\hline Hydromorphone & $1,708 \quad(9.7)$ \\
\hline Morphine & $1,196 \quad(6.8)$ \\
\hline Fentanyl & $319 \quad(1.8)$ \\
\hline Othera & $600 \quad(3.5)$ \\
\hline
\end{tabular}

WOMAC Score 12 Months After TKA. We compared OU (long-term and intermittent) Western Ontario and McMaster Universities Osteoarthritis Index (WOMAC) scores 12 months after TKA to those of opioid-naive patients (reference group) to determine whether the allowable refill gap influences the results of patient outcomes after surgery. The WOMAC is a validated, disease-specific patient-reported outcome instrument commonly used to measure patients' joint pain and function after TKA. ${ }^{35,36}$ Scores ranging from 0-100 are generated for each patient from a Likert scale, with higher scores indicative of better outcome. ${ }^{35}$ Scores stabilize between 6 and 12 months after TKA, and a difference of 10 points on the WOMAC score represents a detectable clinical difference between groups. ${ }^{37}$ Our previous work reported that WOMAC scores after TKA are influenced by opioid use before surgery. ${ }^{17}$

\section{Statistical Analysis}

Medians and interquartile ranges (IQRs) were reported for nonparametric variables, while means and 95\% confidence intervals (CIs) were reported for variables that were normally distributed. Groups were compared using student's t-test for normally distributed variables and the Mann-Whitney test for nonparametric comparisons. Linear regression was performed using the patient's opioid classification as the independent variable and 12-month WOMAC score as the dependent variable. Coefficients were interpreted compared to the defined reference group (opioid-naive patients). Significance was set at $<0.01$. Data preparation and statistics were performed using SAS, version 9.4 (SAS Institute, Cary, NC).

\section{Results}

\section{Study Cohort}

This cohort consisted of 14,252 patients with a mean age of $66.8 \pm 9.3$ years; $60.4 \%$ were female (Table 1). Of these patients, 4,393 patients (31\%) had an opioid prescription in the 180-day period before surgery, with a median number of opioid prescriptions per patient of $3(\mathrm{IQR}=5)$. Of the 17,617 individual opioid prescriptions analyzed, the median length for prescriptions was 25 days $(\mathrm{IQR}=20)$. Codeine $(38.3 \%)$, tramadol (23.9\%), and oxycodone (16.1\%) were the most commonly prescribed opioids (Table 1).

\section{Long-Term Opioid Use}

As the allowable refill gap increased, the proportion of patients classified as an intermittent OU increased, while the number classified as long-term OUs decreased for all 3 methods (Table 2). The number of patients classified as a long-term OUs ranged from 4.4\%-14.6\% (fixed method), 4.2\%-13.2\% (fraction method), and 4.5\%-15.1\% (mixed method), while the number of patients classified as intermittent OUs varied from 26.4\%-16.2\% (fixed method), 26.6\%-17.6\% (fraction method), and $26.4 \%-15.8 \%$ (mixed method; Table 2). This represented a 232\% (fixed method), 214\% (fraction method), and 236\% (mixed method) increase in the number of patients classified as long-term OUs. OU refill gap thresholds were varied from a minimum to maximum (Table 2). Opioid-naive patients remained stable at 69\% for all methods across all refill gap thresholds. Between-method variation (fixed, fraction, and mixed) changed no more than $1.9 \%$ for intermittent OUs and long-term OUs at all fraction or fixed-day refill gap thresholds.

\section{Active Opioid Days in 180 Days Before TKA}

The fixed method minimized the maximum number of days that intermittent OUs held an active opioid prescription in the 180 days before surgery compared with the fraction method (Appendix A, available in online article). At a fraction of 0.5 , the intermittent OU cohort had a patient with an active opioid prescription for 178 days (fraction method) compared with 166 days using the fixed-days method. Again, at a fraction of 1 , the intermittent OU group had a patient who held an active opioid prescription for 162 days compared with 147 days using the fixed methodology. In contrast, the fraction method maximized the minimum number of days that long-term OUs held active opioid prescription in the 180 days before surgery. Long-term OUs had a minimum of 71 days at a fraction of 0.5 compared with 67 days at a refill gap of 14 days. At a fraction of 1 , the long-term OU group had a minimum of 40 days compared with 32 days using the fixed-days method (Appendix A).

As the refill gap increased, the percentage of days longterm OUs held opioid prescriptions in the 180 days before surgery decreased. Long-term OUs held an opioid prescription for $90.2 \%$ of the 180-day window for all 3 methods 


\section{The Influence of Allowable Refill Gaps on Detecting Long-Term Opioid Therapy: An Analysis of Population-Based Administrative Dispensing Data Among Patients with Knee Arthritis Awaiting Total Knee Arthroplasty}

TABLE 2 Comparison of the Prevalence of Opioid Use with Fraction, Fixed, and Mixed Methods

\begin{tabular}{|c|c|c|c|c|c|c|c|c|c|c|c|c|}
\hline \multirow{4}{*}{$\begin{array}{l}\text { Fraction } \\
0.04 \\
\end{array}$} & \multirow{4}{*}{$\begin{array}{c}\text { Fixed } \\
1 \\
\end{array}$} & \multicolumn{5}{|c|}{ Intermittent Opioid User ${ }^{a}$} & \multicolumn{6}{|c|}{ Long-Term Opioid User ${ }^{b}$} \\
\hline & & \multirow{2}{*}{$\begin{array}{c}\text { Mixed }^{c} \\
\%(n)\end{array}$} & \multirow{2}{*}{\multicolumn{2}{|c|}{$\begin{array}{c}\text { Fraction }^{\mathrm{d}} \\
\%(\mathrm{n})\end{array}$}} & \multirow{2}{*}{\multicolumn{2}{|c|}{$\begin{array}{l}\text { Fixed } \\
\%(n) \\
\end{array}$}} & \multirow{2}{*}{\multicolumn{2}{|c|}{$\begin{array}{c}\text { Mixed }^{c} \\
\%(n)\end{array}$}} & \multirow{2}{*}{\multicolumn{2}{|c|}{$\begin{array}{c}\text { Fraction }^{\mathrm{d}} \\
\%(\mathrm{n})\end{array}$}} & \multirow{2}{*}{\multicolumn{2}{|c|}{$\frac{\text { Fixed }}{\%(n)}$}} \\
\hline & & & & & & & & & & & & \\
\hline & & $26.4 \quad(3,758)$ & 26.6 & $(3,792)$ & 26.4 & $(3,763)$ & & (635) & 4.2 & $(601)$ & 4.4 & $(630)$ \\
\hline 0.25 & 7 & $23.8 \quad(3,396)$ & 24.1 & $(3,438)$ & 24.0 & $(3,423)$ & 7.0 & $(997)$ & 6.7 & $(955)$ & 6.8 & $(970)$ \\
\hline 0.5 & 14 & $22.5 \quad(3,210)$ & 22.9 & $(3,263)$ & 23.0 & $(3,278)$ & 8.3 & $(1,183)$ & 7.9 & $(1,130)$ & 7.8 & $(1,115)$ \\
\hline 0.75 & 21 & $21.4 \quad(3,044)$ & 22.0 & $(3,136)$ & 21.9 & $(3,121)$ & 9.5 & $(1,349)$ & 8.8 & $(1,257)$ & 8.9 & $(1,272)$ \\
\hline 1 & 28 & $20.5 \quad(2,927)$ & 21.3 & $(3,035)$ & 21.0 & $(2,998)$ & 10.3 & $(1,466)$ & 9.5 & $(1,358)$ & 9.8 & $(1,395)$ \\
\hline 1.5 & 42 & $19.1 \quad(2,715)$ & 20.2 & $(2,872)$ & 19.6 & $(2,794)$ & 11.8 & $(1,678)$ & 10.7 & $(1,521)$ & 11.2 & $(1,599)$ \\
\hline 2 & 56 & $18.0 \quad(2,558)$ & 19.2 & $(2,740)$ & 18.5 & $(2,636)$ & 12.9 & $(1,835)$ & 11.6 & $(1,653)$ & 12.3 & $(1,757)$ \\
\hline 2.5 & 70 & $17.0 \quad(2,427)$ & 18.6 & $(2,647)$ & 17.5 & $(2,492)$ & 13.8 & $(1,966)$ & 12.3 & $(1,746)$ & 13.3 & $(1,901)$ \\
\hline 3 & 84 & $16.1 \quad(2,297)$ & 17.9 & $(2,551)$ & 16.6 & $(2,365)$ & 14.7 & $(2,096)$ & 12.9 & $(1,842)$ & 14.2 & $(2,028)$ \\
\hline 3.2 & 90 & $15.8 \quad(2,248)$ & 17.6 & $(2,514)$ & 16.2 & $(2,314)$ & 15.1 & $(2,145)$ & 13.2 & $(1,879)$ & 14.6 & $(2,079)$ \\
\hline
\end{tabular}

antermittent $O U$ defined as opioid prescriptions in 180 days before index TKA but does not meet criteria for a long-term OU.

${ }^{b}$ Long-term OU defined as 90 days of consistent opioid use in 180 days before index TKA.

${ }^{c}$ Mixed method selects whichever refill gap is greater (fixed or fraction).

${ }^{d}$ Fraction gap based on percentage length of previous opioid prescription.

$\mathrm{OU}=$ opioid user; $\mathrm{TKA}=$ total knee arthroplasty.

at the minimum allowable refill gap. At the maximum allowable refill gap, long-term OUs held an opioid prescription for 66\% (fixed method), $70.2 \%$ (fraction method), and $65 \%$ (mixed method) of the entire 180-day window before TKA surgery. The difference between the percentage of days that long-term OUs and intermittent OUs held opioid prescriptions in the 180-day period before surgery decreased as the allowable refill gap increased (Appendix A).

\section{Morphine Equivalent Dose}

As the allowable refill gap increased, there was a decrease in median MED for long-term OU classification episodes (Appendix B, available in online article). Median MED decreased 62.3\%, from $77.1 \mathrm{mg}$ MED to $29.3 \mathrm{mg}$ MED (fixed method); decreased 53.5\%, from 74.2 mg MED to $34.5 \mathrm{mg}$ MED (fraction method); and decreased $62.0 \%$, from $75.1 \mathrm{mg}$ MED to $28.2 \mathrm{mg}$ MED (mixed method) as the allowable refill gap varied from minimum to maximum (Appendix B). No intermittent OU had a calculated MED classification episode; none of these patients met the parameters to be included as a long-term OUs.

\section{Effect of Opioid Classification on WOMAC Scores 12 Months After TKA}

The difference in WOMAC scores 12 months after TKA increased from -13.41 to -8.30 (40\%) for long-term OUs when the allowable refill gap increased from 1-90 days compared with opioid-naive patients $(P<0.001$; Table 3$)$. The difference in scores between long-term OUs and opioid-naive patients crossed the threshold that is considered clinically significant after TKA as the allowable refill gap moved from 1-1.5 (fraction method) or 28-42 days (fixed method). Intermittent
OU 12-month WOMAC scores increased from -4.80 to -3.58 points $(17 \%)$ for long-term OUs when the allowable refill gap increased from 1-90 days compared with opioid-naive patients $(P<0.001$; Table 3). Similar trends were observed for the fraction and fixed methods (Table 3).

\section{Discussion}

Despite the growing number of reports outlining rates of opioid use in North America, there is no consensus on how best to define consistent opioid use with administrative pharmaceutical dispensing data. ${ }^{23,29,38,39}$ This has created uncertainty and confusion regarding the actual rates of opioid use in North America among various patient populations, including those with arthritis. ${ }^{14,15,22,40}$ Reported rates of LTOT among patients with hip or knee arthritis awaiting surgery have varied from $5 \%-40 \%$ and are likely not only due to variation in regional opioid prescribing practices but also the parameters used to define opioid exposure. Consistent with these reports, 31\% of patients were dispensed opioids within 180 days of TKA in our study, but the rate of LTOT was dependent on the allowable refill gap. The allowable refill gap also influenced the groups' calculated daily opioid dose, opioid utilization episode mean duration, and whether the difference in outcome scores between long-term OU and opioid-naive patients after TKA was considered clinically significant.

Developing standardized methods to detect consistent opioid use with pharmaceutical dispensing data that align with parameters of LTOT is of great interest, as this threshold is associated with increased rates of adverse events and underlying physiological changes. ${ }^{41,42}$ Long-term exogenous opioid exposure has been reported to change the endogenous opioid 


\section{The Influence of Allowable Refill Gaps on Detecting Long-Term Opioid Therapy: An Analysis of Population-Based Administrative Dispensing Data Among Patients with Knee Arthritis Awaiting Total Knee Arthroplasty}

\section{TABLE 3 Impact of Opioid Classification on 12-Month WOMAC Scores Using Different Refill Gaps}

\begin{tabular}{|c|c|c|c|c|c|c|c|c|c|}
\hline \multirow[b]{2}{*}{ Fraction $^{c}$} & \multirow[b]{2}{*}{ Fixed $^{c}$} & \multicolumn{4}{|c|}{ Opioid Exposed ${ }^{a}$} & \multicolumn{4}{|c|}{ Opioid User ${ }^{b}$} \\
\hline & & $\mathrm{n}$ & Estimate & SE & $P$ Value & $\mathbf{n}$ & Estimate & SE & $P$ Value \\
\hline 0.04 & 1 & 3,758 & -4.80 & 0.87 & $<0.0001$ & 635 & -13.41 & 2.19 & $<0.0001$ \\
\hline 0.25 & 7 & 3,396 & -4.17 & 0.90 & $<0.0001$ & 997 & -12.52 & 1.68 & $<0.0001$ \\
\hline 0.5 & 14 & 3,210 & -3.94 & 0.91 & $<0.0001$ & 1,183 & -11.85 & 1.54 & $<0.0001$ \\
\hline 0.75 & 21 & 3,044 & -3.72 & 0.93 & $<0.0001$ & 1,349 & -11.48 & 1.45 & $<0.0001$ \\
\hline 1 & 28 & 2,927 & -3.90 & 0.94 & $<0.0001$ & 1,466 & -10.23 & 1.38 & $<0.0001$ \\
\hline 1.5 & 42 & 2,715 & -3.76 & 0.97 & 0.0001 & 1,678 & -9.52 & 1.28 & $<0.0001$ \\
\hline 2 & 56 & 2,558 & -3.66 & 1.00 & 0.0002 & 1,835 & -9.07 & 1.22 & $<0.0001$ \\
\hline 2.5 & 70 & 2,427 & -3.54 & 1.02 & 0.0005 & 1,966 & -8.84 & 1.18 & $<0.0001$ \\
\hline 3 & 84 & 2,297 & -3.62 & 1.04 & 0.0005 & 2,096 & -8.36 & 1.14 & $<0.0001$ \\
\hline 3.2 & 90 & 2,248 & -3.58 & 1.05 & 0.0006 & 2,145 & -8.30 & 1.13 & $<0.0001$ \\
\hline \multicolumn{10}{|l|}{ Fraction $^{\mathrm{d}}$} \\
\hline 0.04 & & 3,792 & -4.90 & 0.86 & $<0.0001$ & 601 & -12.95 & 2.24 & $<0.0001$ \\
\hline 0.25 & & 3,438 & -4.30 & 0.89 & $<0.0001$ & 955 & -12.33 & 1.72 & $<0.0001$ \\
\hline 0.5 & & 3,263 & -3.97 & 0.91 & $<0.0001$ & 1,130 & -12.09 & 1.57 & $<0.0001$ \\
\hline 0.75 & & 3,136 & -3.56 & 0.92 & 0.0001 & 1,257 & -12.65 & 1.50 & $<0.0001$ \\
\hline 1 & & 3,035 & -3.65 & 0.93 & $<0.0001$ & 1,358 & -11.51 & 1.43 & $<0.0001$ \\
\hline 1.5 & & 2,872 & -3.76 & 0.95 & $<0.0001$ & 1,521 & -10.25 & 1.35 & $<0.0001$ \\
\hline 2 & & 2,740 & -3.34 & 0.97 & 0.0006 & 1,653 & -10.40 & 1.28 & $<0.0001$ \\
\hline 2.5 & & 2,647 & -3.33 & 0.98 & 0.0007 & 1,746 & -10.03 & 1.25 & $<0.0001$ \\
\hline 3 & & 2,551 & -3.46 & 0.99 & 0.0005 & 1,842 & -9.43 & 1.22 & $<0.0001$ \\
\hline \multirow[t]{12}{*}{3.2} & & 2,514 & -3.57 & 1.00 & 0.0004 & 1,879 & -9.06 & 1.20 & $<0.0001$ \\
\hline & Fixed & & & & & & & & \\
\hline & 1 & 3,763 & -4.80 & 0.87 & $<0.0001$ & 630 & -13.41 & 2.19 & $<0.0001$ \\
\hline & 7 & 3,423 & -4.13 & 0.89 & $<0.0001$ & 970 & -12.99 & 1.71 & $<0.0001$ \\
\hline & 14 & 3,278 & -4.17 & 0.91 & $<0.0001$ & 1,115 & -11.46 & 1.58 & $<0.0001$ \\
\hline & 21 & 3,121 & -3.83 & 0.92 & $<0.0001$ & 1,272 & -11.48 & 1.47 & $<0.0001$ \\
\hline & 28 & 2,998 & -3.94 & 0.94 & $<0.0001$ & 1,395 & -10.40 & 1.41 & $<0.0001$ \\
\hline & 42 & 2,794 & -3.99 & 0.96 & $<0.0001$ & 1,599 & -9.31 & 1.31 & $<0.0001$ \\
\hline & 56 & 2,636 & -4.10 & 0.98 & $<0.0001$ & 1,757 & -8.58 & 1.25 & $<0.0001$ \\
\hline & 70 & 2,492 & -3.90 & 1.00 & 0.0001 & 1,901 & -8.50 & 1.20 & $<0.0001$ \\
\hline & 84 & 2,365 & -4.05 & 1.03 & $<0.0001$ & 2,028 & -7.99 & 1.17 & $<0.0001$ \\
\hline & 90 & 2,314 & -4.00 & 1.03 & 0.0001 & 2,079 & -7.96 & 1.15 & $<0.0001$ \\
\hline
\end{tabular}

\footnotetext{
Note: Reference group is opioid-naive patients.
}

aOpioid exposed defined as opioid prescriptions in 180 days before index TKA but does not meet criteria for an OU.

${ }^{b}$ Opioid user defined as 90 days of consistent opioid use in 180 days before index TKA.

${ }^{c}$ Mixed method selects whichever refill gap is greater (fixed or fraction).

${ }^{d}$ Fraction gap based on percentage length of previous opioid prescription.

$O U=$ opioid user; $S E=$ standard error; TKA = total knee arthroplasty; WOMAC=Western Ontario and McMaster Universities Osteoarthritis Index

system in regions of the brain that regulate both patient's emotions and perception of chronic pain. ${ }^{41}$ It is thought that change to the endogenous opioid system provides a framework to understand why certain high-risk patients with chronic pain, such as those with a history of arthritis and depression, selfselect for LTOT ${ }^{43}$ These patients with a negative affect state are much more likely to transition from short-term to long-term opioid therapy at a higher dose compared with patients who do not have a history of depression or related conditions. ${ }^{44}$ More work is needed to clarify this complex relationship to further understand these high-risk patients, which may help reduce the number of inappropriate opioid prescriptions, in keeping with current opioid North American prescribing guidelines. ${ }^{41}$ The current study will inform future work, since it is based on established parameters for LTOT and adaptable to any pharmaceutical database that contains dispensing dates and prescription durations. ${ }^{20,21,24,45}$

Our approach will enable investigators to determine rates of LTOT using consistent definitions, which will facilitate comparisons of opioid use among different patient populations. Unifying methods that define LTOT have the potential to clarify regional prescribing patterns, the effectiveness of opioid prescribing programs, and variances among different patient populations. For example, Hadlandsmyth et al. (2018) reported 

Method (0.5)

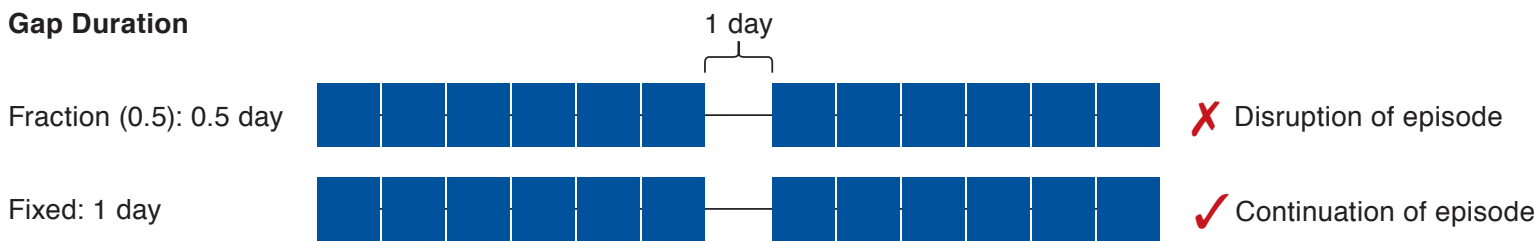

that $20 \%$ of patients were long-term OUs before receiving a TKA in the United States using an allowable refill gap of 14 days with a fixed-day method. ${ }^{20}$ In comparison, only $8 \%$ of patients were long-term OUs among our Canadian cohort using the same refill gap method. This is the first report we are aware of that compares rates of LTOT in this patient population between Canada and the United States using a similar case definition and suggests that, despite similar opioid prescribing guidelines, Canada may have lower rates of LTOT among this patient population. ${ }^{9,10}$ However, these results should be interpreted with caution, as the fixed method was adapted to opioid prescriptions without considering how opioid-specific prescribing practices might affect the classification of long-term OUs.

In other clinical areas, the fixed method of determining refill gaps has been criticized as a static approach that does not consider the length of patient-specific prescriptions. ${ }^{27}$ These limitations in the fixed method were also noted when applied to opioid prescriptions in our cohort. Particularly, when applied to short-term opioid prescriptions, some patients were classified as long-term OUs despite having more days without than with an active opioid prescription (Appendix A). In cardiovascular persistence medication studies, the fraction method appears to provide a better estimate of consistent medication use, as each allowable refill gap is relative to the preceding prescription. ${ }^{27}$ However, when applied to opioid prescriptions, we found that despite circumventing the fixed-method limitations, missing a single day can disrupt the opioid classification episode at a threshold of $<1$ when daily dispensing of opioids is encountered (Figure 2).

This limitation in the fraction approach can misclassify long-term high-dose patients who are often dispensed daily opioids to reduce the risk for opioid misuse and overdose. ${ }^{9}$ In our results, a patient who was prescribed opioids for 178 days of a possible 180 days was classified as an intermittent OU at fractions < 1 (Appendix A). To circumvent the limitations of both approaches for opioid prescriptions, we developed a mixed method that calculated both fixed and fraction methods and defaulted to whichever refill gap was greatest (Figure 1). This method overcame the fraction methodological limitations and maximized the difference between intermittent and long-term OU mean episode duration and daily opioid dose (Appendix A).

A strength of our study was the ability to describe the influence of allowable refill gaps on the detection of LTOT using different methods and relate these changes to a validated patientreported outcome measure, where scores have previously been shown to be influenced by opioid use..$^{17,36}$ We also identified a subgroup of patients (intermittent OUs) who had distinctive characteristics and outcomes from both long-term OUs and opioid-naive patients; these patients were previously excluded in other analyses. ${ }^{30,45}$ Analysis of these 2 distinct groups of OUs highlighted the importance of the 90-day threshold for LTOT. Patients who had consistent opioid dispensing for at least 90 days utilized opioids more frequently and at higher daily doses compared with intermittent OUs. These findings are consistent with reports that suggest that if patients use opioids for longer that 90 days, they tend to use opioids indefinitely. ${ }^{21,46,47}$

\section{Limitations}

This study has some limitations to consider. One limitation was the assumption that opioid dispensing is a surrogate for patient consumption. Multiple methods have been used to determine opioid consumption, including urine or blood tests, patientreported use, or refill data. ${ }^{48}$ While blood serum levels or urine tests are expensive and logistically complex if a large number of patients are required, patient-reported opioid use typically underreports actual opioid use rates. ${ }^{49,50}$ In a study of patients awaiting TKA, patients underreported opioid use by as much as $46 \%$ compared with an opioid monitoring database..$^{50}$

While our study was restricted to patients from Alberta, our provincial-wide database is likely representative of a general population of patients with symptomatic arthritis waiting for TKA, since Canada provides universal health care to all residents and our findings were not restricted to 1 center or surgeon. ${ }^{45}$ Additional research in other settings and disease states would help clarify the generalizability of our study results to determine if these effects are consistent in other prescribing environments or patient populations.

Finally, we were not able to establish an allowable refill gap that best predicts LTOT. Nevertheless, we believe that our 


\section{The Influence of Allowable Refill Gaps on Detecting Long-Term Opioid Therapy: An Analysis of Population-Based Administrative Dispensing Data Among Patients with Knee Arthritis Awaiting Total Knee Arthroplasty}

detailed analysis and described methodology provide valuable information regarding the effect of various allowable refill gaps and different methods that can be used to define LTOT to allow more consistent definition of opioid use and ensure that comparisons across groups are based on clear definitions.

\section{Conclusions}

A significant number of patients with symptomatic knee arthritis were dispensed opioids before TKA, but the allowable refill gap influenced the estimated rate of LTOT. The allowable refill gap also influenced long-term OUs' calculated daily opioid dose, mean opioid utilization episode, and whether clinically significant differences were achieved after TKA when compared with opioid-naive patients. These findings underscore the importance of carefully considering the methods and allowable refill gaps before designing or interpreting study results that examine consistent opioid use. Future work that relates these algorithms to clinical metrics, such as physiciandiagnosed LTOT or LTOT complications, would help clarify the sensitivity and specificity of various refill gap thresholds.

\section{Authors}

C. MICHAEL GOPLEN, MD, Department of Surgery; C. ALLYSON JONES, PT, PhD, School of Public Health and Department of Physical Therapy; LAUREN A. BEAUPRE, PT, PhD, Department of Surgery and Department of Physical Therapy, University of Alberta, Edmonton, Canada. JASON R. RANDALL, PhD; FATEMEH VAKILIAN, MSc; and DONALD C. VOAKLANDER, PhD, School of Public Health, University of Alberta, Edmonton, Canada. SUNG HYUN KANG, MSc, Alberta Bone and Joint Institute, Calgary, Alberta, Canada.

AUTHOR CORRESPONDENCE: C. Michael Goplen, MD, Department of Surgery, University of Alberta, Edmonton, AB, Canada, T6G 2B7. Tel.: 780-394-9264; E-mail: cgoplen@ualberta.ca.

\section{DISCLOSURES}

This work was supported by the Department of Surgery's Clinical Research Grant at the University of Alberta (RES0039945). The authors have no potential conflicts of interest.

\section{REFERENCES}

1. Gomes T, Juurlink DN, Dhalla IA, Mailis-Gagnon A, Paterson JM, Mamdani MM. Trends in opioid use and dosing among socio-economically disadvantaged patients. Open Med. 2011;5(1):e13-22.

2. Wright EA, Katz JN, Abrams S, Solomon DH, Losina E. Trends in prescription of opioids from 2003-2009 in persons with knee osteoarthritis. Arthritis Care Res (Hoboken). 2014;66(10):1489-95.

3. Kenan K, Mack K, Paulozzi L. Trends in prescriptions for oxycodone and other commonly used opioids in the United States, 2000-2010. Open Med. 2012;6(2):e41-7.
4. Canadian Institute for Health Information. Pan-Canadian Trends in the Prescribing of Opioids, 2012 to 2016. Ottawa, ON: CIHI; 2017. Available at: https://secure.cihi.ca/free_products/pan-canadian-trends-opioid-prescribing2017-en-web.pdf. Accessed August 20, 2019.

5. Fischer B, Rehm J, Tyndall M. Effective Canadian policy to reduce harms from prescription opioids: learning from past failures. CMAJ. 2016;188 (17-18):1240-44.

6. Chou R, Fanciullo GJ, Fine PG, et al. Clinical guidelines for the use of chronic opioid therapy in chronic noncancer pain. J Pain. 2009;10(2): 113-130.e22.

7. Boudreau D, Von Korff M, Rutter CM, et al. Trends in long-term opioid therapy for chronic non-cancer pain. Pharmacoepidemiol Drug Saf. 2009;18(12):1166-75

8. Bedson J, Chen Y, Hayward RA, et al. Trends in long-term opioid prescribing in primary care patients with musculoskeletal conditions. Pain. 2016;157(7):1525-31

9. Busse JW. The 2017 Canadian guideline for opioids for chronic non-cancer pain. Natl Pain Cent. 2017:1-105.

10. Dowell D, TM H, Chou R. CDC guideline for prescribing opioids for chronic pain-United States, 2016. JAMA. 2016;315(15):1624-45.

11. American Academy of Orthopedic Surgeons. Opioid use, misuse, and abuse in orthopaedic practice. October 2015. Available at: https:// www.aaos.org/uploadedFiles/PreProduction/About/Opinion_Statements/ advistmt/1045\%20Opioid\%20Use,\%20Misuse,\%20and\%20Abuse $\% 20$ in\%20 Practice.pdf. Accessed August 17, 2019.

12. Busse JW, Wang L, Kamaleldin M, et al. Opioids for chronic noncancer pain. JAMA. 2018;320(23):2448.

13. Krebs EE, Gravely A, Nugent S, et al. Effect of opioid vs nonopioid medications on pain-related function in patients with chronic back pain or hip or knee osteoarthritis pain: the SPACE randomized clinical trial. JAMA 2018;319(9):872-82

14. Ben-Ari A, Chansky H, Rozet I. Preoperative opioid use is associated with early revision after total knee arthroplasty: a study of male patients treated in the Veterans Affairs system. J Bone Jt Surg. 2017;99(1):1-9.

15. Smith SR, Bido J, Collins JE, Yang H, Katz JN, Losina E. Impact of preoperative opioid use on total knee arthroplasty outcomes. J Bone Jt Surgery Am. 2017;99(10):803-08.

16. Morris BJ, Sciascia AD, Jacobs CA, Edwards TB. Preoperative opioid use associated with worse outcomes after anatomic shoulder arthroplasty. J Shoulder Elb Surg. 2016;25(4):619-23.

17. Goplen CM, Verbeek W, Kang SH, et al. Preoperative opioid use is associated with worse patient outcomes after total joint arthroplasty: a systematic review and meta-analysis. BMC Musculoskelet Disord. 2019;20(1):234.

18. Hansen CA, Inacio MCS, Pratt NL, Roughead EE, Graves SE. Chronic use of opioids before and after total knee arthroplasty: a retrospective cohort study. J Arthroplasty. 2017;32(3):817.el.

19. Saïdi H, Pagé MG, Boulanger A, Ware MA, Choinière M. Effectiveness of long-term opioid therapy among chronic non-cancer pain patients attending multidisciplinary pain treatment clinics: a Quebec Pain Registry study. Can J Pain. 2018;2(1):113-24.

20. Hadlandsmyth K, Vander Weg MW, McCoy KD, Mosher HJ, VaughanSarrazin MS, Lund BC. Risk for prolonged opioid use following total knee arthroplasty in veterans. J Arthroplasty. 2018;33(1):119-23.

21. Von Korff M, Saunders K, Thomas Ray G, et al. De facto long-term opioid therapy for noncancer pain. Clin J Pain. 2008;24(6):521-27.

22. Inacio MCS, Hansen C, Pratt NL, Graves SE, Roughead EE. Risk factors for persistent and new chronic opioid use in patients undergoing total hip arthroplasty: a retrospective cohort study. BMJ Open. 2016;6(4).

23. Vanderlip ER, Sullivan MD, Edlund MJ, et al. National study of discontinuation of long-term opioid therapy among veterans. Pain. 2014;155(12):2673-79. 


\section{The Influence of Allowable Refill Gaps on Detecting Long-Term Opioid Therapy: An Analysis of Population-Based Administrative Dispensing Data Among Patients with Knee Arthritis Awaiting Total Knee Arthroplasty}

24. Aroke H, Buchanan A, Wen X, Ragosta P, Koziol J, Kogut S. Estimating the direct costs of outpatient opioid prescriptions: a retrospective analysis of data from the Rhode Island Prescription Drug Monitoring Program. J Manag Care Spec Pharm. 2018;24(3):214-24. Available at: https://www.jmcp.org/ doi/10.18553/jmcp.2018.24.3.214.

25. Gomes T, Mamdani MM, Paterson JM, Dhalla IA, Juurlink DN. Trends in high-dose opioid prescribing in Canada. Can Fam Physician. 2014;60(9):826-32.

26. Vink NM, Klungel OH, Stolk RP, Denig P. Comparison of various measures for assessing medication refill adherence using prescription data. Pharmacoepidemiol Drug Saf. 2009;18(2):159-65.

27. Van Wijk BLG, Klungel OH, Heerdink ER, de Boer A. Refill persistence with chronic medication assessed from a pharmacy database was influenced by method of calculation. J Clin Epidemiol. 2006;59(1):11-17.

28. Parker MM, Moffet HH, Adams A, Karter AJ. An algorithm to identify medication nonpersistence using electronic pharmacy databases. J Am Med Inform Assoc. 2015;22(5):957-61.

29. Gressler LE, Martin BC, Hudson TJ, Painter JT. The relationship between concomitant benzodiazepine-opioid use and adverse outcomes among U.S. veterans. Pain. 2017;159(3):1.

30. Landsman-Blumberg PB, Katz N, Gajria K, Coutinho AD, Yeung PP, White R. Burden of alcohol abuse or dependence among long-term opioid users with chronic noncancer pain. J Manag Care Spec Pharm. 2017;23(7):718-24. Available at: https://www.jmcp.org/doi/full/10.18553/ jmcp.2017.23.7.718.

31. Canadian Institute for Health Information. Wait times for priority procedures in Canada. 2017. Available at: https://www.cihi.ca/en/wait-times-forpriority-procedures-in-canada. Accessed August 20, 2019

32. von Elm E, Altman DG, Egger M, Pocock SJ, Gotzsche PC, Vandenbroucke JP. The Strengthening the Reporting of Observational Studies in Epidemiology (STROBE) statement: guidelines for reporting observational studies. J Clin Epidemiol. 2008;61(4):344-49.

33. Kaur AD, McQueen A, Jan S. Opioid drug utilization and cost outcomes associated with the use of buprenorphine-naloxone in patients with a history of prescription opioid use. J Manag Care Spec Pharm. 2008;14(2):186-94 Available at: https://www.jmcp.org/doi/abs/10.18553/jmcp.2008.14.2.186.

34. Work Safe BC. Claims with opioids, sedative/hypnotics or other prescribed potentially addictive drugs. 2017. Available at: https://www.worksafebc.com/en/resources/law-policy/compensation-practice-directives/claimswith-opioids-sedativehypnotics-or-other-prescribed-potentially-addictive-dru gs?lang=en\&origin=s\&returnurl=https\%3A\%2F\%2Fwww.worksafebc. com\%2Fen\%2Fsearch\%23q\%3DOpioi. Accessed August 17, 2019.

35. Collins NJ, Roos EM. Patient-reported outcomes for total hip and knee arthroplasty: commonly used instruments and attributes of a "good" measure. Clin Geriatr Med. 2012;28(3):367-94.
36. Bellamy N, Buchanan WW, Goldsmith CH, Campbell J, Stitt LW. Validation study of WOMAC: a health status instrument for measuring clinically important patient relevant outcomes to antirheumatic drug therapy in patients with osteoarthritis of the hip or knee. J Rheumatol. 1988;15(12):1833-40

37. Clement ND, Bardgett M, Weir D, Holland J, Gerrand C, Deehan DJ. What is the minimum clinically important difference for the WOMAC Index after TKA? Clin Orthop Relat Res. 2018;476(10):2005-14

38. Calcaterra SL, Scarbro S, Hull ML, Forber AD, Binswanger IA, Colborn KL. Prediction of future chronic opioid use among hospitalized patients. J Gen Intern Med. 2018;33(6):898-905.

39. Kim KY, Anoushiravani AA, Chen KK, Roof M, Long WJ, Schwarzkopf R. Preoperative chronic opioid users in total knee arthroplasty-which patients persistently abuse opiates following surgery? J Arthroplasty. 2017:6-11.

40. Bedard NA, DeMik DE, Dowdle SB, Callaghan JJ. Trends and risk factors for prolonged opioid use after unicompartmental knee arthroplasty. Bone Joint J. 2018;100-B(1 Suppl A):62-67.

41. Sullivan MD. Depression effects on long-term prescription opioid use, abuse, and addiction. 2018;34(9):878-84.

42. Els C, Jackson TD, Kunyk D, et al. Adverse events associated with mediumand long-term use of opioids for chronic non-cancer pain: an overview of Cochrane Reviews. Cochrane Database Syst Rev. 2017;10:CD012509.

43. Sullivan MD. Why does depression promote long-term opioid use? Pain. 2016;157(11):2395-96.

44. Halbert BT, Davis RB, Wee CC. Disproportionate longer-term opioid use among U.S. adults with mood disorders. Pain. 2016;157(11):2452-57.

45. Bedard NA, Pugely AJ, Westermann RW, Duchman KR, Glass NA, Callaghan JJ. Opioid use after total knee arthroplasty: trends and risk factors for prolonged use. J Arthroplasty. 2017;32(8):2390-94.

46. Martin BC, Fan M-Y, Edlund MJ, Devries A, Braden JB, Sullivan MD. Long-term chronic opioid therapy discontinuation rates from the TROUP study. J Gen Intern Med. 2011;26(12):1450-57.

47. Braden JB, Fan M-Y, Edlund MJ, Martin BC, DeVries A, Sullivan MD. Trends in use of opioids by noncancer pain type 2000-2005 among Arkansas Medicaid and HealthCore enrollees: results from the TROUP study. J Pain. 2008;9(11):1026-35.

48. Osterberg L, Blaschke T. Adherence to medication. N Engl J Med. 2005;353(5):487-97.

49. El Khoury AC, Karlawish J, Andrews E, Caplan A. Bioethical issues in pharmacoepidemiologic research. In: Strom L, Kimmel S, Hennessy S, eds Pharmacoepidemiology, Fifth Edition. Hoboken, NJ: Wiley \& Sons; 2012:623-39.

50. Hereford TE, Cryar KA, Edwards PK, Siegel ER, Barnes CL, Mears SC. Patients with hip or knee arthritis underreport narcotic usage. J Arthroplasty. 2018;33(10):3113-17. 


\section{The Influence of Allowable Refill Gaps on Detecting Long-Term Opioid Therapy: An Analysis of Population-Based Administrative Dispensing Data Among Patients with Knee Arthritis Awaiting Total Knee Arthroplasty}

\section{APPENDIX A Duration of Opioid Utilization (Days) Before Index Procedure Based on Different Refill Gaps}

\begin{tabular}{|c|c|c|c|c|c|c|c|c|c|c|c|c|c|c|c|c|}
\hline \multicolumn{2}{|l|}{ Mixeda $^{a}$} & \multicolumn{7}{|c|}{ Intermittent Opioid Userb } & \multicolumn{8}{|c|}{ Long-Term Opioid Userc } \\
\hline Fraction $^{\mathrm{d}}$ & Fixed $^{a}$ & $\mathrm{n}$ & Mean & Min & Max & $95 \mathrm{~L}$ & $95 \mathrm{U}$ & $\begin{array}{c}\text { Utilizatione }^{\mathrm{e}} \\
\%\end{array}$ & $\mathrm{n}$ & Mean & Min & Max & $95 \mathrm{~L}$ & $95 \mathrm{U}$ & $\begin{array}{c}\text { Utilization } \\
\%\end{array}$ & $P$ Value \\
\hline 0.04 & 1 & 3,758 & 50.3 & 1.0 & 178.0 & 48.8 & 51.9 & 28.0 & 635 & 162.5 & 95.0 & 180.0 & 160.7 & 164.2 & 90.3 & $<0.001$ \\
\hline 0.25 & 7 & 3,396 & 39.5 & 1.0 & 164.0 & 38.3 & 40.8 & 22.0 & 997 & 158.6 & 83.0 & 180.0 & 157.1 & 160.0 & 88.1 & $<0.001$ \\
\hline 0.5 & 14 & 3,210 & 34.9 & 1.0 & 162.0 & 33.7 & 36.0 & 19.4 & 1,183 & 152.6 & 67.0 & 180.0 & 151.0 & 154.1 & 84.8 & $<0.001$ \\
\hline 0.75 & 21 & 3,044 & 31.0 & 1.0 & 155.0 & 29.9 & 32.0 & 17.2 & 1,349 & 146.8 & 40.0 & 180.0 & 145.2 & 148.5 & 81.6 & $<0.001$ \\
\hline 1 & 28 & 2,927 & 28.5 & 1.0 & 147.0 & 27.5 & 29.4 & 15.8 & 1,466 & 142.5 & 32.0 & 180.0 & 140.8 & 144.3 & 79.2 & $<0.001$ \\
\hline 1.5 & 42 & 2,715 & 24.8 & 1.0 & 133.0 & 24.0 & 25.7 & 13.8 & 1,678 & 134.1 & 21.0 & 180.0 & 132.2 & 136.0 & 74.5 & $<0.001$ \\
\hline 2 & 56 & 2,558 & 22.5 & 1.0 & 119.0 & 21.7 & 23.3 & 12.5 & 1,835 & 128.0 & 13.0 & 180.0 & 126.0 & 129.9 & 71.1 & $<0.001$ \\
\hline 2.5 & 70 & 2,427 & 20.9 & 1.0 & 104.0 & 20.2 & 21.7 & 11.6 & 1,966 & 122.9 & 6.0 & 180.0 & 120.8 & 124.9 & 68.3 & $<0.001$ \\
\hline 3 & 84 & 2,297 & 19.7 & 1.0 & 93.0 & 19.0 & 20.5 & 11.0 & 2,096 & 117.9 & 1.0 & 180.0 & 115.7 & 120.0 & 65.5 & $<0.001$ \\
\hline 3.2 & 90 & 2,248 & 19.3 & 1.0 & 90.0 & 18.6 & 20.0 & 10.7 & 2,145 & 116.1 & 1.0 & 180.0 & 113.9 & 118.2 & 64.5 & $<0.001$ \\
\hline \multicolumn{17}{|l|}{ Fraction $^{\mathrm{d}}$} \\
\hline 0.04 & & 3,792 & 51.4 & 1.0 & 180.0 & 50.5 & 50.5 & 28.5 & 601 & 162.3 & 95.0 & 180.0 & 160.5 & 164.1 & 90.2 & $<0.001$ \\
\hline 0.25 & & 3,438 & 40.7 & 1.0 & 178.0 & 39.4 & 42.1 & 22.6 & 955 & 159.4 & 83.0 & 180.0 & 158.0 & 160.9 & 88.6 & $<0.001$ \\
\hline 0.5 & & 3,263 & 36.1 & 1.0 & 178.0 & 34.9 & 37.2 & 20.0 & 1,130 & 154.6 & 71.0 & 180.0 & 153.1 & 156.1 & 85.9 & $<0.001$ \\
\hline 0.75 & & 3,136 & 32.9 & 1.0 & 178.0 & 31.9 & 34.0 & 18.3 & 1,257 & 150.4 & 50.0 & 180.0 & 148.9 & 152.0 & 83.6 & $<0.001$ \\
\hline 1 & & 3,035 & 30.6 & 1.0 & 162.0 & 29.6 & 31.6 & 17.0 & 1,358 & 146.9 & 50.0 & 180.0 & 145.3 & 148.5 & 81.6 & $<0.001$ \\
\hline 1.5 & & 2,872 & 27.3 & 1.0 & 154.0 & 26.4 & 28.2 & 15.2 & 1,521 & 140.7 & 40.0 & 180.0 & 139.0 & 142.4 & 78.2 & $<0.001$ \\
\hline 2 & & 2,740 & 24.9 & 1.0 & 154.0 & 24.0 & 25.7 & 13.8 & 1,653 & 135.6 & 13.0 & 180.0 & 133.8 & 137.5 & 75.4 & $<0.001$ \\
\hline 2.5 & & 2,647 & 23.5 & 1.0 & 154.0 & 22.7 & 24.3 & 13.0 & 1,746 & 131.9 & 6.0 & 180.0 & 130.0 & 133.8 & 73.3 & $<0.001$ \\
\hline 3 & & 2,551 & 22.2 & 1.0 & 131.0 & 21.5 & 23.0 & 12.3 & 1,842 & 127.9 & 1.0 & 180.0 & 126.0 & 129.9 & 71.1 & $<0.001$ \\
\hline \multirow[t]{12}{*}{3.2} & & 2,514 & 21.8 & 1.0 & 131.0 & 21.0 & 22.5 & 12.1 & 1,879 & 126.5 & 1.0 & 180.0 & 124.5 & 128.4 & 70.3 & $<0.001$ \\
\hline & \multicolumn{16}{|l|}{ Fixed } \\
\hline & 1 & 3,763 & 50.5 & 1.0 & 178.0 & 48.9 & 52.1 & 28.1 & 630 & 162.4 & 95.0 & 180.0 & 160.7 & 164.2 & 90.2 & $<0.001$ \\
\hline & 7 & 3,423 & 40.3 & 1.0 & 166.0 & 39.0 & 41.6 & 22.4 & 970 & 159.3 & 87.0 & 180.0 & 157.9 & 160.7 & 88.5 & $<0.001$ \\
\hline & 14 & 3,278 & 36.6 & 1.0 & 166.0 & 35.4 & 37.8 & 20.3 & 1,115 & 154.6 & 67.0 & 180.0 & 153.1 & 156.2 & 85.9 & $<0.001$ \\
\hline & 21 & 3,121 & 33.0 & 1.0 & 158.0 & 31.9 & 34.1 & 18.4 & 1,272 & 148.8 & 40.0 & 180.0 & 147.1 & 150.4 & 82.7 & $<0.001$ \\
\hline & 28 & 2,998 & 30.4 & 1.0 & 147.0 & 29.3 & 31.4 & 16.9 & 1,395 & 144.3 & 32.0 & 180.0 & 142.6 & 146.0 & 80.2 & $<0.001$ \\
\hline & 42 & 2,794 & 26.7 & 1.0 & 135.0 & 25.8 & 27.7 & 14.8 & 1,599 & 136.2 & 21.0 & 180.0 & 134.2 & 138.1 & 75.6 & $<0.001$ \\
\hline & 56 & 2,636 & 24.2 & 1.0 & 124.0 & 23.3 & 25.1 & 13.4 & 1,757 & 130.1 & 13.0 & 180.0 & 128.1 & 132.1 & 72.3 & $<0.001$ \\
\hline & 70 & 2,492 & 22.1 & 1.0 & 110.0 & 21.3 & 22.9 & 12.3 & 1,901 & 124.8 & 9.0 & 180.0 & 122.7 & 126.8 & 69.3 & $<0.001$ \\
\hline & 84 & 2,365 & 20.8 & 1.0 & 96.0 & 20.0 & 122.1 & 11.5 & 2,028 & 119.9 & 7.0 & 180.0 & 117.8 & 122.1 & 66.6 & $<0.001$ \\
\hline & 90 & 2,314 & 20.1 & 1.0 & 90.0 & 19.4 & 20.9 & 11.2 & 2,079 & 118.2 & 6.0 & 180.0 & 116.1 & 120.3 & 65.7 & $<0.001$ \\
\hline \multicolumn{17}{|c|}{$\begin{array}{l}\text { a Mixed method selects whichever refill gap is greater (fixed or fraction). } \\
\text { bIntermittent OU defined as opioid prescriptions in } 180 \text { days before index TKA but does not meet criteria for a long-term OU. } \\
\text { cLong-term OU defined as } 90 \text { days of consistent opioid use in } 180 \text { days before index TKA. } \\
{ }^{d} \text { Fraction gap based on percentage length of previous opioid prescription. } \\
\text { eP value calculated with 2-sample Students t-test comparing median dose between } 2 \text { groups. } \\
95 \mathrm{~L}=95 \% \text { confidence lower limit; } 95 U=95 \% \text { confidence upper limit; IQR=interquartile range; Min=minimum; Max=maximum. }\end{array}$} \\
\hline
\end{tabular}



The Influence of Allowable Refill Gaps on Detecting Long-Term Opioid Therapy: An Analysis of Population-Based
Administrative Dispensing Data Among Patients with Knee Arthritis Awaiting Total Knee Arthroplasty

\begin{tabular}{|c|c|c|c|c|c|c|c|c|c|}
\hline \multicolumn{2}{|c|}{ APPENDIX B } & $\begin{array}{l}\text { ated D } \\
\text { ant Re }\end{array}$ & $\begin{array}{l}\text { lorphi } \\
\text { os }\end{array}$ & quivaler & Dose In & l) for & lassifica & Episor & ed on \\
\hline \multicolumn{2}{|l|}{ Mixed $^{a}$} & \multicolumn{8}{|c|}{ Long-Term Opioid Userb } \\
\hline Fraction $^{\mathrm{c}}$ & Fixed & $\mathrm{n}$ & Mean & Median & IQR & Min & Max & $95 \mathrm{~L}$ & $95 \mathrm{U}$ \\
\hline 0.04 & 1 & 635 & 151.9 & 75.1 & 125.6 & 4.5 & $3,200.0$ & 133.0 & 170.8 \\
\hline 0.25 & 7 & 997 & 122.5 & 64.0 & 96.9 & 4.4 & $2,827.6$ & 110.4 & 134.6 \\
\hline 0.5 & 14 & 1,183 & 106.7 & 58.5 & 82.1 & 2.5 & $2,620.1$ & 96.7 & 116.7 \\
\hline 0.75 & 21 & 1,349 & 97.2 & 52.4 & 70.9 & 2.5 & $2,620.1$ & 88.4 & 106.0 \\
\hline 1 & 28 & 1,466 & 91.2 & 47.0 & 66.5 & 2.5 & $2,620.1$ & 83.0 & 99.3 \\
\hline 1.5 & 42 & 1,678 & 81.8 & 40.6 & 60.1 & 2.8 & $2,458.1$ & 74.8 & 88.9 \\
\hline 2 & 56 & 1,835 & 76.3 & 36.1 & 53.6 & 2.1 & $2,458.1$ & 69.7 & 82.9 \\
\hline 2.5 & 70 & 1,966 & 71.6 & 32.1 & 50.1 & 1.9 & $2,458.1$ & 65.5 & 77.8 \\
\hline 3 & 84 & 2,096 & 67.5 & 29.3 & 47.3 & 1.7 & $2,458.1$ & 61.7 & 73.3 \\
\hline 3.2 & 90 & 2,145 & 66.2 & 28.3 & 46.8 & 1.5 & $2,458.1$ & 60.5 & 71.9 \\
\hline \multicolumn{10}{|l|}{ Fractionc } \\
\hline 0.04 & & 601 & 147.5 & 74.2 & 126.5 & 4.5 & $3,200.0$ & 129.2 & 165.9 \\
\hline 0.25 & & 955 & 124.5 & 64.7 & 99.4 & 4.4 & $2,827.6$ & 111.9 & 137.1 \\
\hline 0.5 & & 1,130 & 109.5 & 59.2 & 86.2 & 2.5 & $2,620.1$ & 99.0 & 119.9 \\
\hline 0.75 & & 1,257 & 101.6 & 54.8 & 77.1 & 2.5 & $2,620.1$ & 92.2 & 111.0 \\
\hline 1 & & 1,358 & 95.5 & 50.1 & 72.9 & 2.5 & $2,620.1$ & 86.8 & 104.2 \\
\hline 1.5 & & 1,521 & 88.1 & 44.0 & 65.9 & 2.8 & $2,620.1$ & 80.2 & 96.0 \\
\hline 2 & & 1,653 & 83.1 & 40.8 & 60.7 & 2.1 & $2,458.1$ & 75.9 & 90.4 \\
\hline 2.5 & & 1,746 & 79.5 & 38.1 & 56.3 & 1.9 & $2,458.1$ & 72.6 & 86.4 \\
\hline 3 & & 1,842 & 75.6 & 35.2 & 53.2 & 1.7 & $2,458.1$ & 69.1 & 82.2 \\
\hline \multirow[t]{12}{*}{3.2} & & 1,879 & 74.5 & 34.5 & 52.1 & 1.7 & $2,458.1$ & 68.0 & 80.9 \\
\hline & Fixed & & & & & & & & \\
\hline & 1 & 630 & 153.3 & 77.1 & 125.5 & 4.5 & $3,200.0$ & 134.1 & 172.4 \\
\hline & 7 & 970 & 125.7 & 65.5 & 98.5 & 4.4 & $2,827.6$ & 113.2 & 138.2 \\
\hline & 14 & 1,115 & 112.6 & 60.3 & 87.8 & 4.4 & $2,620.1$ & 101.9 & 123.2 \\
\hline & 21 & 1,272 & 102.3 & 55.4 & 75.0 & 3.9 & $2,620.1$ & 93.0 & 111.7 \\
\hline & 28 & 1,395 & 95.3 & 50.5 & 68.4 & 3.9 & $2,620.1$ & 86.8 & 103.9 \\
\hline & 42 & 1,599 & 85.1 & 42.1 & 62.2 & 3.1 & $2,458.1$ & 77.7 & 92.5 \\
\hline & 56 & 1,757 & 78.9 & 37.5 & 55.9 & 2.9 & $2,458.1$ & 72.1 & 85.7 \\
\hline & 70 & 1,901 & 73.9 & 33.5 & 52.0 & 2.5 & $2,458.1$ & 67.6 & 80.2 \\
\hline & 84 & 2,028 & 69.9 & 30.5 & 49.3 & 2.3 & $2,458.1$ & 63.9 & 75.9 \\
\hline & 90 & 2,079 & 68.2 & 29.3 & 47.8 & 1.5 & $2,458.1$ & 62.4 & 74.1 \\
\hline \multicolumn{10}{|c|}{$\begin{array}{l}{ }^{a} \text { Mixed method selects whichever refill gap is greater (fixed or fraction). } \\
{ }^{b} \text { Long-term OU defined as } 90 \text { days of consistent opioid use in } 180 \text { days before index TKA. } \\
\text { cFraction gap based on percentage length of previous opioid prescription. } \\
95 \mathrm{~L}=95 \% \text { confidence lower limit; } 95 \mathrm{U}=95 \% \text { confidence upper limit; } I Q R=\text { interquartile rar }\end{array}$} \\
\hline
\end{tabular}

